Cuadernos de Filología Clásica. Estudios Latinos

ISSN: 1131-9062

http://dx.doi.org/10.5209/CFCL.56191

\title{
El uso de argumentos de la filosofía clásica contra el paraíso carnal de Mahoma $^{1}$
}

\author{
David Vázquez Ruiz ${ }^{2}$
}

Recibido: 30 de marzo de 2016 / Aceptado: 21 de julio de 2016

Resumen. En 1687 vio la luz la obra bajo el título Manuductio ad conuersionem Mahumetanorum del padre Tirso González de Santalla (1624-1705), quien ese mismo año obtuvo el cargo como decimotercer Prepósito General de la Compañía de Jesús. La obra, siguiendo la estela de la literatura de controversia interreligiosa medieval, se nutre de diversos autores cuya retórica y método llevan siglos utilizándose para combatir el Islam, y que, en última instancia, podríamos registrar en los primeros trabajos de carácter polémico contra judíos y paganos. Aquí, sin embargo, podemos encontrar directamente la palabra de los clásicos utilizada, cristianamente -eso sí-, para reprobar las costumbres y el modo de vida de los seguidores de Mahoma. El objetivo fundamental del presente escrito es ofrecer una muestra del uso de argumentos de la filosofía clásica de autores como Aristóteles o Séneca (entre otros), en la refutación contra la vida del Islam desde un punto de vista carnal y moral.

Palabras clave: Manuductio; Tirso González; paraíso; filosofía; carnal.

\section{[en] The use of arguments from classical philosophy against Muhammad's carnal paradise}

\begin{abstract}
In 1687 was published the work called Manuductio ad conuersionem Mahumetanorum by pater Tirso González de Santalla (1624-1705), who in that same year achieved the tittle of Superior General of the Society of Jesus. That work -continuing the line of the medieval controversy interreligious literature- receives from a group of authors whose rhetoric and method were been using during centuries to combat Islam, and -at the end- we can register them in the firsts works of polemic against Jews and pagans. Here, nevertheless, we can directly find the word of classics used-Christian way- to reprove customs and the way of life of the followers of Muhammad. The fundamental object of the present work is an example of use of arguments from classical authors like Aristotle or Seneca (among others), in the refutation against the Islam life from a carnal and righteous point of view.
\end{abstract}

Key words: Manuductio; Tirso González; paradise; philosophy; carnal.

Sumario: 1. Introducción. 2. Exempla. 3. Reflexiones finales. 4. Referencias bibliográficas.

Cómo citar: Vázquez Ruiz, D., «El uso de argumentos de la filosofía clásica contra el paraíso carnal de Mahoma», Cuad. Filol. Clásc. Estud. Lat. 37.1 (2017), 129-136.

\footnotetext{
1 Una primera versión del trabajo fue presentada como comunicación bajo el mismo título en el IIIr Congreso Nacional Ganimedes. Organizado por la Asociación Ganimedes de Investigadores Noveles de Filología Clásica. Celebrado en Vitoria-Gasteiz entre los días 11 y 13 de marzo de 2015.

2 Universidad Autónoma de Barcelona. Dpto. de Ciencias de la Antigüedad y la Edad Media. Filología Latina.

E-mail:.david.vazquez.ruiz@gmail.com
} 


\section{Introducción}

A finales del siglo XVII proliferará la aparición de ciertas obras de carácter polemista y de controversia tanto contra musulmanes, como judíos y herejes, en la península ibérica y alrededores de Europa, en gran parte de la mano de la Compañía de Jesús. En mayor medida, este hecho se vio potenciado por la derrota del ejército Otomano a las puertas de Viena en el año 1683, por parte del emperador Leopoldo de Habsburgo quien estaba a cargo de esta Liga Santa ${ }^{3}$.

Tras el detonante, las misiones y obras llevadas a cabo por jesuitas que habían dedicado su vida a la predicación y las misiones, en especial contra el mundo islámico, dieron como resultado una vía de actuación paralela en esta guerra santa, que no implicaba el uso directo de las armas, sino el de la tinta y la pluma, dedicada a refutar el Islam a través del estudio del mismo ${ }^{4}$. No es nada nuevo, sin embargo, este tipo de lucha, ya venía perfeccionándose, transmitiéndose y reelaborándose, desde las primeras traducciones del Corán y los textos islámicos en la península ibérica allá por el siglo XII (Martínez Gázquez 2003; 2005). No obstante, aquí encontraremos un grupo de jesuitas dispuestos a la elaboración de ciertos 'manuales' para la conversión (Colombo 2009a, 315-318) (dentro de la literatura de controversia interreligiosa que abarcaba desde sermones y catecismos, hasta diálogos entre cristianos y musulmanes $^{5}$ ), siendo algunos de ellos Paolo Segneri ${ }^{6}$, Nicolò Pallavicino ${ }^{7}$ o Michel Nau .

En este contexto, Tirso González de Santalla (1624-1705) teólogo y jesuita con dos décadas de experiencia como misionero a lo largo y ancho del panorama peninsular, y que llegaría a ser nombrado Prepósito General de la Compañía ${ }^{9}$, decidió

3 El segundo sitio de Viena o batalla de Kahlenberg que tuvo lugar el 11 y 12 de septiembre de 1683 supondría el inicio del declive para el Imperio Otomano en Europa. Tras la victoria de Leopoldo I de Habsburgo, rey de Hungría y Bohemia, y Emperador del Sacro Imperio Romano Germánico desde 1658, a la cabeza de un ejército coaligado junto a la Mancomunidad de Polonia y Lituania (Liga Santa), se produjo en el territorio cristiano europeo una corriente de pensamiento dispuesta a interpretar de forma profética el significado de dicho triunfo, visto como la victoria del cristianismo sobre el Islam. Esta idea está presente en Colombo (2009a y 2012).

4 Para un extenso catálogo de obras y trabajos de carácter polemista y literatura de refutación latina medieval en general, $\mathrm{c} f$. proyecto de investigación del Departamento de Ciencias de la Antigüedad y la Edad Media (UAB) Islamolatina. La percepción del Islam en la Europa latina, dirigido actualmente por la profesora Cándida Ferrero Hernández.

5 Para un interesante muestrario de obras y trabajos latinos de carácter polemista hasta el siglo XV, cf. Ferrero 2011.

6 Paolo Segneri $(† 1694)$ teólogo, polemista y predicador italiano, llegaría a predicador personal del Papa Inocencio XII. Escribió una obra sobre la fe de la iglesia con diversas referencias islámicas bajo el título: L’incredulo senza scusa, dove si dimostra che non poù non conoscere quale sia la vera Religione, chi vuol conoscerla (1690).

7 Nicolò Pallavicino $(† 1692)$ teólogo personal de la reina Cristina de Suecia, realizó diversas obras de carácter polemista: Le moderne prosperità della Chiesa Cattolicacontro il Maccomettismo, in cui si dimostra la cura usata da Dio col Cristianesimo contro i Turchi, e si commendano que 'potentati, e Duci, che hanno formata la Sagra Lega, o sono concorsi ad essa: mostrando ai primi la necessità di continuarla, e ad altri di intraprenderla, con dare a vedere l'obbligo, che hanno i cristiani di concorrere a distruggere l'Imperio Ottomano (1688); L'evidente merito della fede cattolica ad essere creduta per vera. In cui si dimostra la verità di quelli articoli, che sono fondamento non solo della vera Religione, ma di qualunque Religione (1689); y La grandezza della Madre di Dio contro le moderne eresie, in cui si rifiutano le antiche e moderne eresie contro la Divina Maternità, e le altre Doti della Vergine (1690).

8 Michel Nau $(\dagger 1683)$ nacido en Tours, dedicó casi veinte años a las misiones en Siria. Escribió: Religio Christiana contra Alcoranum per Alcoranum pacifice defensa ac provata (1680); y L'état présent de la religión mahométane, contenant le choses, les plus curieuses qui regardent Mahomet et l'établissement de la secte (1684).

9 Para una información más extensa sobre el padre Tirso González, cf. Pinedo (2001, 1644-1650) y Reyero (1913, 1-25). 
recoger sus conocimientos misioneros ab experientia authoris-como él mismo señala ${ }^{10}$ - en 1687 en su obra Manuductio ad conuersionem Mahumetanorum ${ }^{11}$, actualmente objeto de estudio de mi tesis doctoral.

Este trabajo publicado en Madrid presenta dos partes, la primera in quo sensibilibus argumentis Diuinitas Christi Domini euidenter demonstratur et euidens credibilitas Religionis Christianae proponitur; y la segunda, in qua falsitas Mahumetanae sectae conuincitur pariterque ueritas Religionis Christianae, quae prima parte demonstrata est a nobis iterum argumentis stabilitur ${ }^{12}$. Esta última supone un verdadero manual para la formación de misioneros dispuestos a entregarse a la evangelización, refutación y conversión, así como la renovación espiritual de los oyentes. Esta fórmula de enseñanza supone una continuación de la retórica y método empleados por diversas fuentes medievales que han seguido la estela de los clásicos latinos en última instancia (Barkai 1984 y 1994; Martínez Gázquez 2003 y 2005; Ferrero 2011; Platas 2011), es decir, nos encontramos ante argumentos ya conocidos pero que han ido repitiéndose y reelaborándose a lo largo de distintos periodos ya desde las primeras obras contra paganos y judíos (Sánchez Salor 1986), ahora -eso si- especialmente aplicados contra musulmanes.

Llegado este punto, podemos observar algunos usos de argumentos de la filosofía clásica mediante tópicos moralizantes (en mayor medida a través de citaciones directas y máximas) en esta obra del padre González. Para ello, he seleccionado un fragmento que ofrece una buena cantidad de ejemplos interesantes. Corresponde al capítulo final (número 11) del segundo de los libros de la segunda parte, que aparece con el siguiente comentario del contenido: Liber secundus. In quo continentur apertae aliquae demonstrationes contra hanc sectam, quae confirmantur ex mirabilibus quorundam Mahumetanorum conuersionibus, specialiter uero falsitas Alcorani conuincitur ex eo quod ponat ultimam foelicitatem hominis in delectationibus corporis. (Md. II, 2, p.57). La última premisa, eso sí, pasa a recogerse expresamente en el mencionado capítulo 11: Caput XI. Conuincitur falsitatis sectae Mahumetanae ex eo quod ponat hominis beatitudinem in delectationibus carnalibus, gustus et tactus. (Md. II, 2, p.126).

Sintetizando nuestra búsqueda, dentro del capítulo aparecen los subapartados 46 y $47^{13}$, en los que el autor nos explica cómo la búsqueda de la verdadera felicidad del hombre ya ha sido estudiada por los antiguos filósofos. Sobre ello, Tirso da tres grandes premisas: la primera centrada en Epicuro y sus seguidores, quienes anteponen los placeres del cuerpo como culmen de la felicidad del hombre. La segunda,

10 Dentro del proemio a la edición de 1687 en Madrid: specialiter in hac secunda parte placuit proponere argumenta illa quibus saepe usus sum ad expugnandam Mahumetanorum perfidiam, cum ad illos publice conciones haberem in pluribus Hispaniae ciuitatibus, nimirum Malaga, Velez-Malaga, Maruella, Gibraltar, Xerez de la Frontera, Puerto de Sancta Maria, Cadiz, San Lucar de Barrameda, Hispali, Ronda, Arcos, et in Curia Matritensi. Nam his argumentis, gratia adspirante diuina, in his locis ducenti permoti sunt ad abiurandum Mahumetismum, et Christianam fidem suscipiendam.

11 Título original: Manuductio ad conuersionem Mahumetanorum, in duas partes diuisa. In prima, Veritas Religionis Christianae Catholicae Romanae manifestis argumentis demonstratur. In secunda, Falsitas Mahumetanae Sectae conuincitur. Siempre que hablemos de la obra nos referiremos a ella como Manuductio o manual, dejando de lado alguna otra denominación. Del mismo modo las citas tomarán por base la primera edición de 1687, impresa en Madrid, mediante la abreviatura Md., seguida de la parte (I o II), el libro, capítulo -si fuera necesario- y finalmente la página.

12 Para algunos estudios enfocados directamente sobre la obra, $c f$. Colombo (2007) y Vázquez (2015a y 2015b).

13 Para facilitar el seguimiento temático de los capítulos de la obra, la impresión de 1689 ofrece estos puntos numerados que respeto e incluyo en mi edición. 
perteneciente a los estoicos, que resume en la máxima uiuere conuenienter naturae et rationi. Y finalmente, la tercera y más correcta para nuestro autor que corresponde a Aristóteles, al considerar la mayor felicidad del hombre la contemplación de Dios y lo divino.

$<46>$. Maximae olim inter Philosophos extitere concertationes circa finem hominis, uidelicet qua in re consistat hominis beatitudo, quodque sit summum eius bonum. Tres autem hac de re fuerunt sententiae celebriores. Prima, summum hominis bonum consistere in uoluptatibus corporis. Haec passim tribuitur Epicuro, eiusque sectatoribus. Secunda, summum hominis bonum consistere in uirtute, siue in eo, quod est uiuere conuenienter naturae et rationi. Ita Stoici. Tertia Aristotelis, qui constituit beatitudinem potissimum in perfectissima operatione praestantissimae facultatis circa praestantissimum obiectum, hoc est in comtemplatione Dei et rerum diuinarum quantum fas est homini. (Md. II, 2, p.133).

Después, en el punto 47 aclara de qué modo, al seguir Mahoma la primera de las opciones y no solo en el ámbito carnal sino también espiritual, está incluso aún más equivocado de lo que ya lo estaba Epicuro y sentencia finalmente in illa non potest consistere hominis beatitudo.

$<47>$. Ex his tribus Philosophorum sententiis prima Epicuri et sequacium contemnitur a cordatioribus Philosophis; et nihilominus illam sectatus est Mahumetus et quidem absurdius, quam Epicurus. Nam iste licet posuerit foelicitatem in uoluptate non tamen in uoluptate solius corporis, sed in uoluptate corporis et animi et utrasque uoluit esse moderatas. At Mahumetus in solis uoluptatibus corporis illisque immoderatissimis beatitudinem et foelicitatem hominis collocat. Vnde eius sententia est absurdissima et contra lumen rationis. Cum enim homo sit animal rationale et ab omnibus brutis differat essentialiter non potest consistere eius foelicitas et beatitudo in illis operationibus, quas habet communes cum brutis: delectatio autem quae capitur ex cibo et potu et ex usu ueneris communis est homini cum brutis; ergo in illa non potest consistere hominis beatitude. (Md. II, 2, p.134).

A partir de este punto comienzan a aparecer distintos ejemplos de los cuales hemos seleccionado algunos para su presentación y comentario.

\section{Exempla}

En el primero de los ejemplos que presentamos, se menciona a Aristóteles y su Historia Animalium (Hist.an.5.2.540b), mediante el comentario ofrecido de los elefantes y los camellos que se esconden tras la cópula, casi como si sintiesen algún tipo de vergüenza; Tirso concluye, ¿cómo es posible sostener la felicidad del hombre ante una actitud tan impúdica?

Si camelus et elephas ut refert Aristoteles lib. 5 de Histor Anim. cap. 2 latibulum quaerunt ad coitum et elephas post coitum iterum absconditur, quasi facti eum pu- 
deat et mox se abluit, quasi sordes contraxerit; quomodo beatitudo hominis potest in tam pudendo opere consistere?. (Md. II, 2, p.136).

En el segundo vemos un ejemplo del filósofo Séneca (Epist.65.21), con la máxima «Mayor soy y para mayores cosas he nacido que para ser esclavo de mi carne» ${ }^{14}$ :

Si Seneca, cum esset Philosophus Gentilis, serio dicebat Epist. 65 Maior sum et ad maiora natus quam ut corporis mei mancipium sim, quaerendo scilicet corporis delectationes et gulae et ueneri indulgendo. (Md. II, 2, p.137).

El ejemplo de Sócrates, el tercero, se resume en la sentencia: «Otros viven para comer, yo como para vivir ${ }^{15}$. Haciendo así referencia a la necesidad de moderarse para conseguir la deseada aurea mediocritas:

Si Socrates interrogatus in quo ipse differret ab aliis hominibus qui delectationes gustus immoderate quaerebant respondit: Alii uiuunt ut edant, ego edo ut uiuam. (Md. II, 2, p.137).

El cuarto ejemplo es de Horacio (Epist.1.2.27). Contiene, además, un error de Tirso, pues indica el segundo libro en su cita, referenciándola a Lelium, cuando debería ser Lollium) y se sirve de la máxima: «Somos estadística, nacidos para consumir los frutos ${ }^{16}$. Continúa el comentario con un particular ataque:

Si Oratius lib. 2 Epist. 2 ad Lelium homini quem deprimere et humiliare uolebat per opprobrium et contemptum dixit, ipsum ad hoc solum natum esse ut comederet, Nos numerus sumus et fruges consumere nati; quid isti Philosophi dicerent si Mahumetum audirent praedicantem supremam hominis foelicitatem consistere in uentris ingluuie et uenereis delectationibus? Certe dicerent illum non hominum sed pecudum Magistrum appellari debere. (Md. II, 2, p.137).

Los siguientes dos ejemplos se encuentran dentro del subapartado 51, en el que explica la importancia de la futura vida espiritual tras la muerte y utiliza pasajes de Lucas y Mateo (20 y 22 respectivamente ${ }^{17}$ ) para hacer entender la poca importancia que merecen las relaciones terrenales, en este caso con la mujer. De esta manera, después enlaza con la idea malum est mulier, sed necessarium malum y la sentencia de Pitágoras «el fin de la vida es la semejanza de Dios», que utiliza para dar a entender cómo la perfección de una criatura racional consiste en la imitación de su creador:

\footnotetext{
Traducción propia.

Traducción propia.

Traducción de Horacio Silvestre (Cátedra) con modificaciones propias.

O quam longe est ab hac caecitate diuina Christi doctrina, qui Matth. 22. Saducaeis negantibus resurrectionem, et ab incommodo arguentibus, quia si eadem foemina successiue nupsisset septem Viris, in resurrectione non posset determinari, cuiusnam de septem uxor futura esset; Dominus respondit: Erratis nescientes scripturas, et uirtutem Dei. In resurrectione enim, nec nubunt, nec nubentur: sed erunt sicut Angeli Dei in Coelo. Et Luc. 20. Nec nubunt, nec ducunt uxores, qui digni habentur saeculo illo $<\ldots>$ Audiant Mahumetani celebre illud Catonis pronuntiatum qui dixit, Si sine uxoribus mundus esse posset, uitam nostram futuram esse qualis est Deorum Coelestium, quare eas malum esse sed malum necessarium. (Md. II, 2, p.138).
} 
Audiant Mahumetani celebre illud Catonis pronuntiatum qui dixit, Si sine uxoribus mundus esse posset, uitam nostram futuram esse qualis est Deorum Coelestium, quare eas malum esse sed malum necessarium.

Idque cognouit Pithagoras Philosophus Gentilis quando dixit: Vitae finis est Dei assimilation. (Md. II, 2, p.139).

Los dos ejemplos posteriores -séptimo y octavo- son usados para remarcar el error de la búsqueda de la felicidad a través del placer ya que este corrompe al hombre y deteriora su alma, haciéndole caer en el pecado. El séptimo, de Cicerón (Sen.44) nos dice cómo Platón llama al placer el cebo del mal, puesto que los hombres son atrapados por él como los peces por un anzuelo:

Idem testantur etiam Ethnici Scriptores. Cic. in Cat. Diuine Plato escam malorum appellat uoluptatem, quod ea uidelicet homines capiantur ut hamo pisces. (Md. II, 2, p.140).

El octavo, por su parte, corresponde al pasaje addiximus animum uoluptati, cui indulgere initium omnium malorum est, tradimus ambitioni et famae, ceteris aeque uanis et inanibus (SEN.Epist.110.10): «hemos librado nuestra alma a los placeres, la licencia de los cuales es el inicio de todos los males, la hemos abandonado a la ambición y al deseo de la fama y a otras cosas igualmente vanas y vacías» ${ }^{18}$ : Et Seneca Epist. 3 Indulgere uoluptati initium omnium malorum est. (Md. II, 2, p.140).

Un último ejemplo que he querido añadir es el de la máxima horaciana (Epist.1.4.16) contra Albio Tibulo: Epicuri de grege porcus (Md. II, 2, p.141), que Tirso incluye en el mismo fragmento que los dos ejemplos anteriores, mientras continúa su invectiva contra la imposición de los placeres carnales y en general, los placeres de la vida, como forma de alcanzar la felicidad.

\section{Reflexiones finales}

En cierto modo y teniendo en cuenta la facilidad interpretativa que presentan las sentencias, se observaría que su uso no dejar de ser puramente ornamental. La mayoría de los ejemplos corresponden a frases de autores clásicos que acabarán dando lugar a máximas que incluso hoy día utilizamos con la intención de aquello que queremos transmitir. Por ello estos comentarios -cuyos autores resultan en cierto modo fácilmente 'cristianizables'- pasan a formar parte de un listado de ejemplos y argumentos tópicos moralizantes usados por el padre Tirso como arma que pretende ridiculizar el denominado paraíso carnal de Mahoma.

Por otro lado, aunque no es sumamente excepcional, sí es sin embargo llamativo a la hora de estudiar el manuductio, nos encontramos con una forma de citar a los autores clásicos más bien vaga, a diferencia de las citas de obras de autores medievales, y además de literatura polémica, por las que González demuestra una gran minuciosidad; aquí se observan unas referencias más sencillas. Algunos pasajes no están citados directamente y otros son incorrectos. Podríamos apuntar quizá -como

18 Traducción propia. 
hemos recogido en el párrafo anterior- a su mera función como máximas empleadas para refutar a Mahoma y el lugar en que deposita la felicidad del hombre, sabiendo que in illa non potest consistere hominis beatitudo.

\section{Referencias bibliográficas}

Barkai, R. (1984), El enemigo en el espejo. Cristianos y musulmanes en la España medieval, Madrid.

Barkai, R. (1994), «Diálogo filosófico-religioso en el seno de las tres culturas ibéricas», en Santiago-Otero, H. (ed.) Diálogo filosófico-religioso entre el cristianismo, judaísmo e islamismo durante la Edad Media en la península ibérica, Turnhout.

Colombo, E. (2007), Convertire i musulmani. L'esperienza di un jesuita spagnolo del Seicento. Milán.

Colombo, E. (2009a), «Jesuits and Islam in Seventeenth-Century Europe: War, Preaching and Conversions», en Heyberger, B., García-Arenal, M., Colombo, E., Vismara, P. (eds.) L'Islam visto da occidente. Cultura e religione del Seicento europeo di fronte all'Islam, Milán-Génova.

Colombo, E. (2009b), «La compagnia di Gesù e l'evangelizzacione dei Musulmani nella Spagna del seicento: Il caso González», RM n. s., t. 20 (=t. 81), 203-227.

Colombo, E. (2012), «Even among Turks. Tirso González de Santalla (1624-1705) and Islam», Studes in the spirituality of jesuits 44 (3).

Ferrero, C. (2011), «Literatura latina de controversia religiosa en la castilla del siglo XV: una aproximación a su tipología», en De la Cruz, O., Ferrero, C. y Martínez Gázquez, J. (eds.) Estudios de Latín Medieval Hispánico, Actas del V Congreso Internacional de Latín Medieval Hispánico, Florencia, 425-441.

Framiñán de Miguel, Ma J. (2005), «Manuales para el adoctrinamiento de neoconversos en el siglo XVI», Criticón 93, 25-37.

Martínez Gázquez, J. (2003), «Trois traductions médiévales latines du Coran: Pierre le Vénérable - Robert de Ketton, Marc de Tolède et Jean de Segobia», Revue des Études Latines 80, 223-235.

Martínez Gázquez, J. (2005), «Finalidad de la primera traducción latina del Corán», en Barceló, M. y Martínez Gázquez, J. (eds.) Musulmanes y cristianos en Hispania durante las conquistas de los siglos XII y XIII, Bellaterra, 71-77.

Pinedo, I. (2001) «Generales», 13, en O’Neill, C. E.; Domínguez, J. M. (eds.), Diccionario histórico de la Compañia de Jesús: biográfico-temático (4 vols.), Madrid, 1644-ss.

Platas, R. (2011), «Ramón Martí y la renovación de la literatura latina de controversia», en Martínez Gázquez, J. et alii (eds.) Estudios de latín medieval hispánico, Actas del V Congreso Internacional de Latín Medieval Hispánico, Florencia.

Reyero, E. (1913), Misiones del R. M. P. Tirso González de Santalla, Santiago de Compostela.

Ruiz, E. - García-Monje, Ma I. (2003), Confusión o confutación de la secta Mahomética y del Alcorán, Salamanca.

Sánchez Salor, E. (1986), Polémica entre cristianos y paganos a través de los textos: problemas existenciales y problemas vivenciales, Madrid.

Santos Neila, F. (1975), «La moral islámica y Tirso González de Santalla», Revista de Estudios Extremeños 31(2). 
Vázquez, D. (2015a), «Una aproximación al Manuductio ad conuersionem Mahumetanorum de Tirso González de Santalla», Myrtia 30, 215-227.

Vázquez, D. (2015b), «La construcción polémica en el texto Manuductio ad conuersionem Mahumetanorum a partir de fuentes medievales», en Olaya N., Montoza M., Aguilera A., Gómez R. (eds.), II Jornadas Predoctorales en Estudios de la Antigüedad y de la Edad

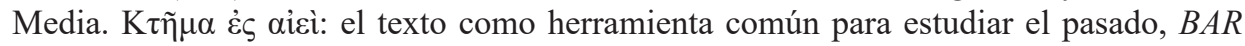
S2775, 197-201. 